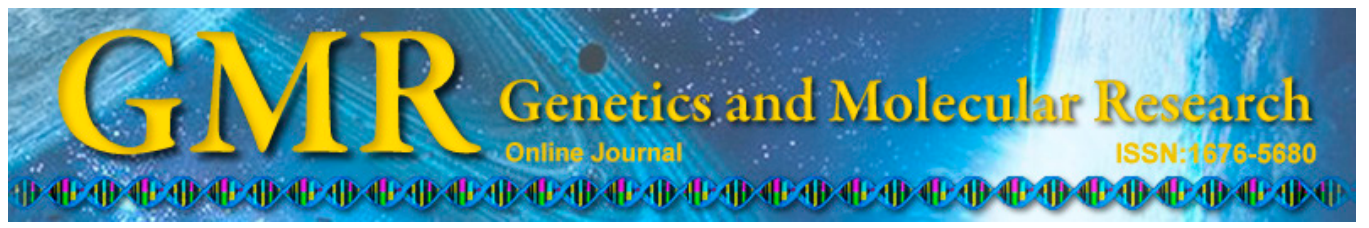

\title{
Genome-wide association study of meat quality traits in chicken
}

\author{
T. Zhang ${ }^{1,2 *}$, Q.C. Fan ${ }^{1,2 *}$, J.Y. Wang ${ }^{1,2}$, G.X. Zhang ${ }^{1,2}$, Y.P. Gu ${ }^{3}$ and \\ Y. Tang ${ }^{1,2}$ \\ ${ }^{1}$ College of Animal Science and Technology, Yangzhou University, \\ Yangzhou, Jiangsu, China \\ ${ }^{2}$ Key Laboratory for Animal Genetics, Breeding, \\ Reproduction and Molecular Design of Province, Jiangsu, \\ Jiangsu Yangzhou, China \\ ${ }^{3}$ Jiangsu Jinghai Industry Poultry Group Co, Ltd., Nantong, Jiangsu, China \\ *These authors contributed equally to this study. \\ Corresponding author: J.Y. Wang \\ E-mail: jywang@yzu.edu.cn
}

Genet. Mol. Res. 14 (3): 10452-10460 (2015)

Received October 14, 2014

Accepted April 24, 2015

Published September 8, 2015

DOI http://dx.doi.org/10.4238/2015.September.8.6

\begin{abstract}
Meat quality traits are very important in the poultry industry. To identify single nucleotide polymorphisms (SNPs) and candidate genes affecting meat quality traits, a genome-wide association study was performed using the Illumina chicken $60 \mathrm{~K}$ SNP beadchip in Jinghai yellow chicken. Four meat quality traits were measured. Two SNPs reached 5\% Bonferroni genome-wide significance $(\mathrm{P}<1.8 \mathrm{E}-6)$ and 7 SNPs reached "suggestive" genomewide significance $(\mathrm{P}<3.59 \mathrm{E}-6)$ with meat quality. These SNPs were located nearby or in 7 candidate genes, including $C B L N 2, H P G D S$, SETD2, and ANKRD46, among others. A total of 5650 haplotpyes were established and only 1 was found to be associated with fat content in leg muscle. These results indicate that the 9 SNPs and
\end{abstract}


7 genes are important candidate markers and may influence meat quality traits in chicken.

Key words: Chicken; Genome-wide association study; Haplotype; Meat quality traits

\section{INTRODUCTION}

Meat quality traits have an important influence on the poultry industry as the flavor of meat is determined by meat quality traits. Many local chicken breeds in China are wellknown for their delicious meat. Remarkable advances in the study of meat quality traits have been achieved in China, and numerous genes and quantitative trait loci have been found to be associated with meat quality traits (Abasht and Lamont, 2007; Atzmon et al., 2008; Fang et al., 2010; Tang et al., 2011).

Polymerase chain reaction-restriction fragment length polymorphism and polymerase chain reaction-single-strand conformation polymorphism are widely used to identify SNPs associated with different traits in chicken. Genome-wide association studies (GWAS) have become one of the most commonly used strategies for identifying genes affecting complex traits in humans and other animals. In chickens, some major loci associated with growth $(\mathrm{Gu}$ et al., 2011; Xie et al., 2012), egg production (Liu et al., 2011; Wolc et al., 2012), rumpless and ear-tufted traits (Noorai et al., 2012), body composition and meat quality (Liu et al., 2013), resistance to Marek's disease, and the immune response to Newcastle disease virus were identified by GWAS (Li et al., 2013; Luo et al., 2013).

In the present study, to identify SNPs and candidate genes that are significantly associated with meat quality traits, a genome-wide association study was conducted using the $60 \mathrm{~K}$ SNP beadchip in Jinghai yellow chicken. We sought to identify new candidate genes and region and lay a foundation for marker-assisted selection of Jinghai yellow chicken.

\section{MATERIAL AND METHODS}

\section{Experimental population and phenotypic measurements}

The population examined in this study included 212 female Jinghai yellow chickens from 19 half-siblings from the same batch. These birds were hatched on the same day and reared in the pens. Birds had access to feed (commercial corn-soybean diets meeting the National Research Council's requirements) and water ad libitum. All chickens were in good health. Blood samples were collected when the chickens were slaughtered at 66 weeks of age. Samples of breast muscle and leg muscle were taken to detect the fat content in breast muscle and leg muscle (FLM) as well as the protein content in breast muscle and leg muscle. The descriptive statistics of the traits above are shown in Table 1. The date was transformed using Johnson transformation to follow the standard normal distribution in Minitap (v16).

\section{Genotyping and quality control}

DNAs were extracted using the Dzup Genomic DNA Isolation Reagent (Blood) kit 
from Sangon Biotech (Shanghai, China). The concentration and quality of genomic DNA was quantified by spectrophotometry and then stored at $-20^{\circ} \mathrm{C}$. DNA samples were sent to DNA Landmark (Québec, Canada) for genotyping using the 60K SNP beadchip.

Plink (v1.07) was used for quality control of the data (Purcell et al., 2007). The samples with low call rate $(<90 \%)$ and SNPs showing low call frequency $(<95 \%)$, low Hardy-Weinberg equilibrium $(<1.0 \mathrm{E}-6)$, and low minor allele frequency $(<3 \%)$ were rejected. Finally, 200 of the 212 samples and 46,665 SNPs were examined in this genome-wide association study. The distribution of SNPs after quality control is shown in Table 2 .

Table 1. Descriptive statistics of carcass and meat quality traits.
\begin{tabular}{lrrrrrr}
\hline Traits & Max & Min & Average & SD & Best-fit & Normal distribution \\
\hline FBM (\%) & 2.8 & 0.8 & 1.3 & 0.3 & 0.65 & Yes \\
FLM (\%) & 4.9 & 2.0 & 3.4 & 0.6 & 0.52 & Yes \\
PBM (\%) & 27.4 & 16.8 & 24.7 & 1.3 & 0.56 & Yes \\
PLM (\%) & 25.4 & 16.5 & 21.7 & 1.0 & 0.68 & Yes \\
\hline
\end{tabular}

Table 2. Basic statistics of small nucleotide polymorphisms after quality control.

\begin{tabular}{|c|c|c|c|}
\hline $\mathrm{Chr}$ & Physical map (Mb) & No. of SNPs & SNP density ${ }^{1}$ \\
\hline 1 & 200.95 & 7244 & 27.74 \\
\hline 2 & 154.79 & 5466 & 28.32 \\
\hline 3 & 113.62 & 4165 & 27.28 \\
\hline 4 & 94.20 & 3396 & 27.74 \\
\hline 5 & 62.23 & 2192 & 28.39 \\
\hline 6 & 35.84 & 1729 & 20.73 \\
\hline 7 & 38.30 & 1829 & 20.94 \\
\hline 8 & 30.56 & 1350 & 22.64 \\
\hline 9 & 24.02 & 1187 & 20.24 \\
\hline 10 & 22.42 & 1302 & 17.22 \\
\hline 11 & 21.87 & 1241 & 17.62 \\
\hline 12 & 20.46 & 1403 & 14.58 \\
\hline 13 & 18.27 & 1186 & 15.41 \\
\hline 14 & 15.76 & 1018 & 15.52 \\
\hline 15 & 12.93 & 1029 & 12.56 \\
\hline 16 & 0.42 & 11 & 37.99 \\
\hline 17 & 10.61 & 846 & 12.55 \\
\hline 18 & 10.85 & 857 & 12.66 \\
\hline 19 & 9.90 & 830 & 11.93 \\
\hline 20 & 13.92 & 1484 & 9.38 \\
\hline 21 & 6.86 & 766 & 8.95 \\
\hline 22 & 3.90 & 300 & 12.99 \\
\hline 23 & 6.02 & 601 & 10.02 \\
\hline 24 & 6.38 & 743 & 8.58 \\
\hline 25 & 2.02 & 168 & 12.00 \\
\hline 26 & 5.07 & 640 & 7.82 \\
\hline 27 & 4.83 & 479 & 10.07 \\
\hline 28 & 4.47 & 560 & 7.99 \\
\hline LGE22C19W28_E50C23 & 0.88 & 109 & 8.10 \\
\hline LGE64 - - & 0.018 & 3 & 6.00 \\
\hline $\mathrm{z}$ & 74.58 & 1942 & 38.40 \\
\hline 0 & 0.00 & 589 & 0.00 \\
\hline Total & 1026.948 & 46665 & 22.04 \\
\hline
\end{tabular}

${ }^{1}$ Unit of SNP density was $\mathrm{Kb} / \mathrm{SNP}$. Chr = chromosome. 


\section{Population structure}

\section{Statistics analysis}

Thepopulation structure of the experimental population was evaluated by multidimensional scaling analysis using the Plink program. The steps were as follows: linkage disequilibrium based on SNP pruning of autosomes was applied with an $\mathrm{r}^{2}>0.2$, and 12,877 independent SNPs were obtained. Next, pair-wise identity-by-state distances of the SNPs and multidimensional scaling analysis components were calculated. The multidimensional scaling analysis diagram was plotted using the R (2.15.1) program (Wang et al., 2009). Principal component analysis (PCA) was performed using the genome-wide complex trait analysis software v1.24. PCA1 and PCA2 were used in models to the reduce population stratification effect (Wall et al., 2003; Wang et al., 2009; Ramos et al., 2009). Haplotype analysis was carried out using the PLINK program based on 46,665 SNPs. The method was used to calculate the $\mathrm{R}^{2}$ value of SNPs within a $200-\mathrm{kb}$ window on the same chromosome. If $\mathrm{R}^{2}>0.8$, the 2 SNPs were considered to be linked.

The general linear regression model (GLM, I) in PLINK was used in this study. The following model equation was used:

$$
\mathrm{Y}=\mathrm{G} \alpha+\mathrm{X} \beta+\mathrm{e}
$$

where $Y$ is the vector of observations; $G$ is genetic effect vector, $X$ is a matrix containing all other fixed effects, including population structure (PCA1 and PCA2), $\alpha$ and $\beta$ are incidence matrix, and $e$ is the vector of random residual. Furthermore, the association analysis between haplotypes and meat quality traits was also performed using the GLM. The Bonferroni P value was calculated based on the independent SNPs of autosomes, which were defined using the indep-pairwise option with $\mathrm{r}^{2}=0.4$ (Johnson et al., 2010). Ultimately, 27,824 SNPs were identified. The threshold $\mathrm{P}$ value for Bonferroni potential significance was 3.59E-5 $(1 / 27,824)$, and the P value for Bonferroni genome-wide significance was $1.80 \mathrm{E}-6(0.05 / 27,824)$. QQ plots and Manhattan plots were created using the R (2.15.1) software.

\section{RESULTS}

\section{Population structure}

The result showed that the distribution of 200 chickens from the 19 half-sibling families showed some degree of stratification (Figure 1). The population structure was corrected using principal component analysis (PCA). PCA1 and PCA2 were used in models to reduce the population stratification effect.

\section{Genome-wide association analysis}

Two SNPs showing genome-wide significance $(\mathrm{P}<1.8 \mathrm{E}-6)$ with FLM were identified using the GLM model. Two SNPs, rs312796105 and rs15469825, were located on GGA1 and shared the same proximal gene of LOC101747478 (Table 3). Seven SNPs showing "suggestive" genome-wide significance (3.59E-5) were identified to be associated with FLM, fat content in breast muscle and protein content in leg muscle using the GLM model. Four 
SNPs, including rs313563594, rs313986006, rs14743029, and rs 16136932, were located on GGA2. The rs1z59929, rs15544591, and rs14300880 were located on GGA1, 4, and 26, respectively (Table 4). No significant SNP was found to be associated with protein content in breast muscle. The Manhattan plots for FLM with significant SNPs were drawn using the R (2.15.1) software (Figure 2). A total of 5650 haplotypes were identified based on the 46,665 SNPs. Only 1 haplotype showing "suggestive" genome-wide significance was identified to be associated with FLM. Names of genes in 1-Mb segments surrounding each significant SNP were downloaded from Ensembl and NCBI. Nine SNPs were located in or nearby 7 candidate genes, including LOC101747478, CBLN2, and HPGDS, among others.

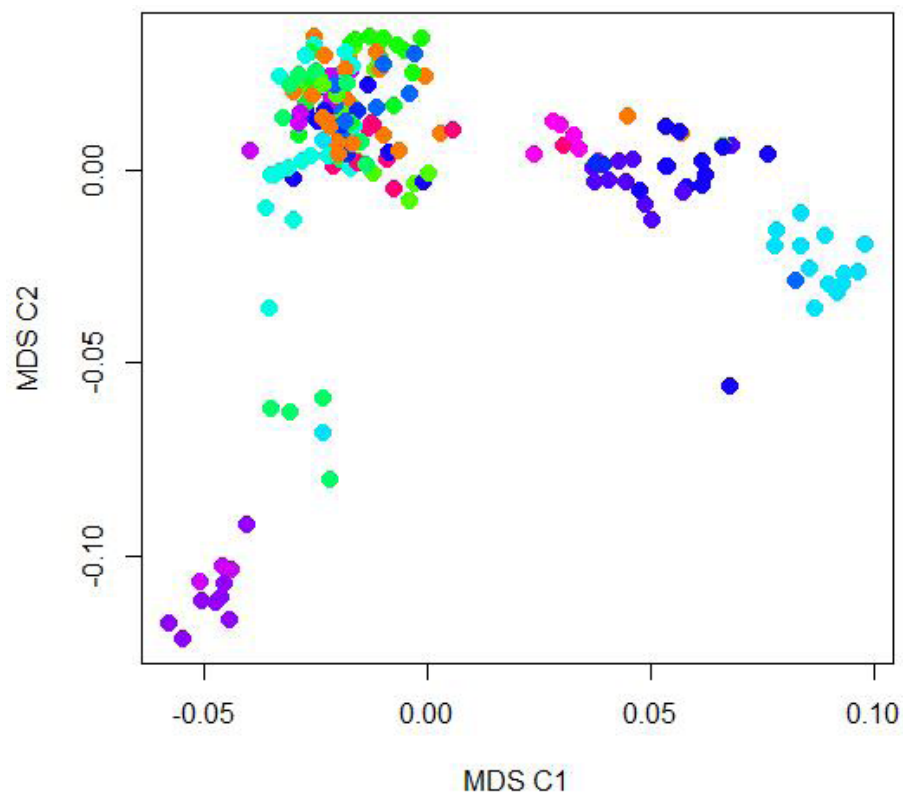

Figure 1. Population structure identified by Multidimensional Scaling analysis.

Table 3. Significant small nucleotide polymorphisms for meat quality traits.

\begin{tabular}{llccccc}
\hline Trait & SNP ID & Chromosome & Position (bp) & P value & Nearest gene & Distance \\
\hline FLM & rs312796105 & 1 & 159933083 & $1.18 \mathrm{E}-06$ & LOC101747478 & $15 \mathrm{~kb}$ downstream \\
& rs15469825 & 1 & 159851745 & $1.60 \mathrm{E}-06$ & LOC101747478 & $56 \mathrm{~kb}$ downstream \\
\hline
\end{tabular}

Table 4. "Suggestive" significant small nucleotide polymorphismsfor meat quality traits.

\begin{tabular}{llcrlll}
\hline & SNP ID & Chromosome & Position $(\mathrm{bp})$ & P value & Nearest gene & Distance \\
\hline FLM & rs313563594 & 2 & 92854994 & $4.85 \mathrm{E}-06$ & CBLN2 & $224.659 \mathrm{~kb}$ upstream \\
& rs1z59929 & 1 & 165000000 & $3.02 \mathrm{E}-05$ & LOC101747478 & $20 \mathrm{~kb}$ downstream \\
& rs15544591 & 4 & 36643430 & $3.10 \mathrm{E}-06$ & HPGDS & $7.362 \mathrm{~kb}$ upstream \\
FBM & rs313986006 & 2 & 3876584 & $2.86 \mathrm{E}-05$ & SETD2 & Within \\
& rs14743029 & 2 & 128320097 & $9.72 \mathrm{E}-06$ & ANKRD46 & $7.910 \mathrm{~kb}$ downstream \\
& rs16136932 & 2 & 130238033 & $2.04 \mathrm{E}-05$ & ZFPM2 & Within \\
PLM & rs14300880 & 26 & 4378338 & $1.50 \mathrm{E}-05$ & GRM4 & $1.814 \mathrm{~kb}$ downstream \\
\hline
\end{tabular}




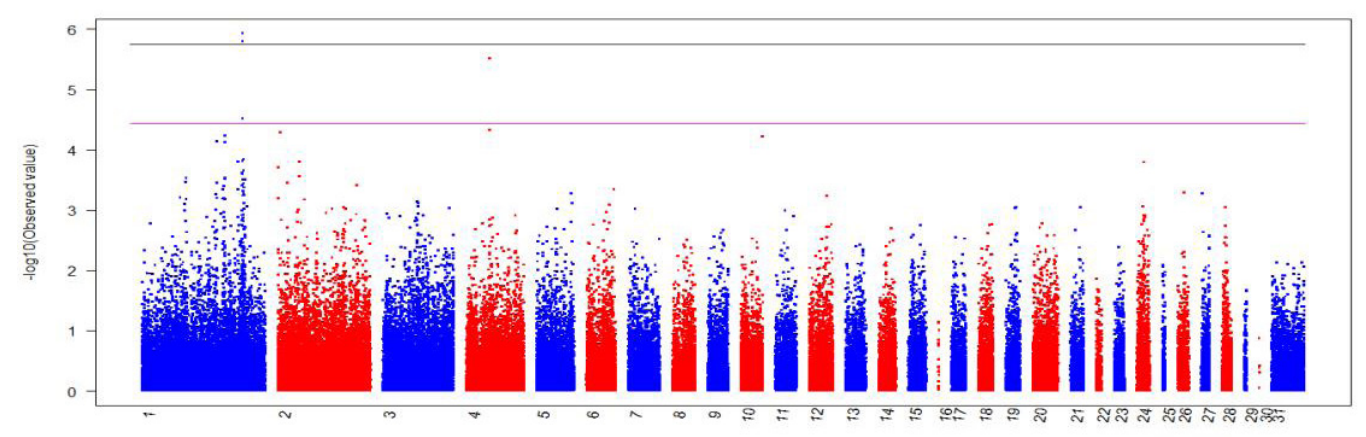

Chromosome

Figure 2. Manhattan plot for genome-wide association study on FLM.

\section{DISCUSSION}

In the present study, 2 SNPs that showed genome-wide significance $(\mathrm{P}<1.8 \mathrm{E}-6)$ with FLM were identified using the GLM model. These 2 SNPs were located on GGA1 and shared the same proximal gene of $L O C 101747478$. Seven SNPs showing "suggestive" genome-wide significance were identified (3.59E-5) using the GLM model. Nine SNPs were located in or nearby 7 different candidate genes.

Three SNPs associated with FLM were located in downstream of the LOC101747478 gene, indicating that LOC101747478 may influence FLM in chickens. LOC101747478 is a novel gene that has not been previously reported. rs313563594 and rs15544591 were also found to be associated with FLM in chicken. They are located upstream of the CBLN2 gene on GGA2 and the HPGDS gene on GGA4. It has been reported that $C B L N 2$ may play a role in synapse formation and in brain evolution (Reiner et al. 2011). CBLN1-null mice are severely ataxic, walk with an irregular gait, and do not maintain their balance on rotarod (Wei et al., 2009). Additionally, there is a high degree of sequence homology between CBLN2 (91\%, based on mouse sequences) and CBLN4 (85\%) with $C B L N 1$, and they may play similar roles in synapse formation.

Hematopoietic prostaglandin D synthase (HPGDS) is mainly expressed in Th-2 cells, mast cells, and antigen-presenting cells. HPGDS can convert prostaglandin $\mathrm{H} 2$ into prostaglandin D2, which is a mediator thought to play a pivotal role in airway allergy and inflammatory processes as well as induce vasodilatation, bronchoconstriction, pulmonary eosinophil and lymphocyte infiltration, and cytokine release in asthmatics (Kanaoka and Urade, 2003). Inhibition of HPGDS may be therapeutically beneficial in the treatment of allergic disease and may be more effective than blocking either DP-1 or chemoattractant receptor-homologous molecules expressed on Th2 cells alone (Trivedi et al., 2006).

SET domain-containing 2 (SETD2) is a histone methyltransferase gene located on 3p21.31. SETD2 is nonredundantly responsible for trimethylation of the histone mark $H 3 K 36$ and is a tumor suppressor gene involved in the development of clear cell renal cell carcinoma (Edmunds et al., 2008; Duns et al., 2010). Li et al. (2005) reported that SETD2 plays a role in chromatin structure modulation during transcriptional elongation via its interaction with hyperphosphorylated POLR2A. It also may act as a transcription activator that binds to promoters. 
Ankyrin repeat domain 46 is a member of ankyrins. Ankyrins are adaptor proteins that attach membrane proteins to the cytoskeleton, and cytoskeletal proteins are important for the oncogenic process of migration and in disease states such as muscular dystrophy (Buscaglia and Li, 2011). Ankyrin repeat domain 46 was identified as a direct target of miR-21 (Cho, 2012). Yan et al. (2011) recently reported that in breast cancer cells, miR-21 is upregulated, corresponding with downregulation of ankyrin repeat domain 46 , leading to increased proliferation and migration.

Zinc finger protein, multitype 2 (ZFPM2) is a target of miR-200 and can regulate the activity of phosphatidylinositol 3-kinase (the upstream activator of Akt) in insulin signaling (Park et al., 2013). Greenbaum et al. (2012) reported that the ZFPM2 SNP rs12678719 was associated with antipsychotic-induced parkinsonism. Tan et al. (2012) reported that variants of the ZFPM2/FOG2 gene may be a common cause of double outlet right ventricle.

Glutamate receptor, metabotropic 4 is a member of the group III metabotropic glutamate receptor genes. Presynaptic metabotropic glutamate receptors, including mGluR4, are known to modulate the release of glutamate and gamma-aminobutyric acid in the thalamocortical network, and perturbations of these pathways have been shown to play a role in several animal models of absence seizures. In particular, disruption of mGluR4 function in mice leads to increased susceptibility to absence seizures. Glutamate receptor, metabotropic 4 sequence variants may confer low-risk effects to the etiology of idiopathic generalized epilepsies. Jiang et al. (2014) reported that polymorphism in the metabotropic glutamate receptor 4 (GRM4) gene were associated with the susceptibility and prognosis of osteosarcoma in a Chinese Han population. In conclusion, all the 7 candidate genes discussed above are novel genes and have not been studied before in chicken before. However, most of those genes are important factors participating in many pathways. And the relationship between those candidate genes and meat quality traits of chicken will be verified in the further study.

\section{CONCLUSIONS}

In the present study, a genome-wide association study was carried out to identify candidate genes associated with meat quality traits in chicken. Nine significant SNPs were detected. Two SNPs, rs312796105, and rs15469825, associated with FLM reached a genomewide significance level. Seven SNPs reached a "suggestive" genome-wide significance level associated with FLM, fat content in breast muscle, and protein content in leg muscle. Seven candidate genes in 1-Mb segments surrounding each significant SNP were obtained. This result indicated that these SNPs and genes are important candidate markers and genes and require further examination.

\section{ACKNOWLEGMENTS}

Research supported by the National Broiler Industrial and Technology System (\#nycytx-42-G1-05), the Priority Academic Program Development of Jiangsu Higher Education Institutions, and the Excellent Doctor Degree Dissertation Foundation of Yangzhou University. 


\section{REFERENCES}

Abasht B and Lamont SJ (2007). Genome-wide association analysis reveals cryptic alleles as an important factor in heterosis for fatness in chicken F2 population. Anim. Genet. 38: 491-498.

Atzmon G, Blum S, Feldman M, Cahaner A, et al. (2008). QTLs detected in a multigenerational resource chicken population. J. Hered. 99: 528-538.

Buscaglia LE and Li Y (2011). Apoptosis and the target genes of microRNA-21. Chin. J. Cancer 30: 371-380.

Cho WC (2012). MicroRNAs as therapeutic targets and their potential applications in cancer therapy. Exp. Opin. Ther. Targets 16: 747-759.

Duns G, van den Berg E, van Duivenbode I, Osinga J, et al. (2010). Histone methyltransferase gene SETD2 is a novel tumor suppressor gene in clear cell renal cell carcinoma. Cancer Res. 70: 4287-4291.

Edmunds JW, Mahadevan LC and Clayton AL (2008). Dynamic histone H3 methylation during gene induction: HYPB/ Setd2 mediates all H3K36 trimethylation. EMBO J. 27: 406-420.

Fang M, Nie Q, Luo C, Zhang D, et al. (2010). Associations of GHSR gene polymorphisms with chicken growth and carcass traits. Mol. Biol. Rep. 37: 423-428.

Greenbaum L, Smith RC, Lorberboym M, Alkelai A, et al. (2012). Association of the ZFPM2 gene with antipsychoticinduced parkinsonism in schizophrenia patients. Psychopharmacology 220: 519-528.

Gu X, Feng C, Ma L, Song C, et al. (2011). Genome-wide association study of body weight in chicken F2 resource population. Plos One 6: e21872.

Jiang C, Chen H, Shao L and Dong Y (2014). GRM4 gene polymorphism is associated with susceptibility and prognosis of osteosarcoma in a Chinese Han population. Med. Oncol. 31: 50.

Johnson RC, Nelson GW, Troyer JL, Lautenberger JA, et al. (2010). Accounting for multiple comparisons in a genomewide association study (GWAS). BMC Genomics 11: 724.

Kanaoka Y and Urade Y (2003). Hematopoietic prostaglandin D synthase. Prostaglandins Leukot. Essent. Fatty Acids 69: 163-167.

Li DF, Lian L, Qu LJ, Chen YM, et al. (2013). A genome-wide SNP scan reveals two loci associated with the chicken resistance to Marek's disease. Anim. Genet. 44: 217-222.

Li M, Phatnani HP, Guan Z, Sage H, et al. (2005). Solution structure of the Set2-Rpb1 interacting domain of human Set2 and its interaction with the hyperphosphorylated C-terminal domain of Rpb1. Proc. Natl. Acad. Sci. U. S. A. 102: 17636-17641.

Liu R, Sun Y, Zhao G, Wang F, et al. (2013). Genome-wide association study identifies loci and candidate genes for body composition and meat quality traits in Beijing-You chickens. Plos One 8: e61172.

Liu W, Li D, Liu J, Chen S, et al. (2011). A genome-wide SNP scan reveals novel loci for egg production and quality traits in white leghorn and brown-egg dwarf layers. PLoS One 6: e28600.

Luo C, Qu H, Ma J, Wang J, et al. (2013). Genome-wide association study of antibody response to Newcastle disease virus in chicken. BMC Genet. 14: 42.

Noorai RE, Freese NH, Wright LM, Chapman SC, et al. (2012). Genome-wide association mapping and identification of candidate genes for the rumpless and ear-tufted traits of the Araucana chicken. PloS One 7: e40974.

Park JT, Kato M, Yuan H, Castro N, et al. (2013). FOG2 protein down-regulation by transforming growth factor- $\beta 1$ induced microRNA-200b/c leads to Akt kinase activation and glomerular mesangial hypertrophy related to diabetic nephropathy. J. Biol. Chem. 288: 22469-22480.

Purcell S, Neale B, Todd-Brown K, Thomas L, et al. (2007). PLINK: a tool set for whole-genome association and population-based linkage analyses. Am. J. Hum. Genet. 81: 559-575.

Ramos AM, Crooijmans RP, Affara NA, Amaral AJ, et al. (2009). Design of a high density SNP genotyping assay in the pig using SNPs identified and characterized by next generation sequencing technology. Plos One 4: e6524.

Reiner A, Yang M, Cagle MC and Honig MG (2011). Localization of cerebellin-2 in late embryonic chicken brain: Implications for a role in synapse formation and for brain evolution. J. Comp. Neurol. 519: 2225-2251.

Tan ZP, Huang C, Xu ZB, Yang JF, et al. (2012). Novel ZFPM2/FOG2 variants in patients with double outlet right ventricle. Clin. Genet. 82: 466-471.

Tang S, Ou J, Sun D, Zhang Y, et al. (2011). A novel 62-bp indel mutation in the promoter region of transforming growth factor-beta 2 (TGFB2) gene is associated with body weight in chickens. Anim. Genet. 42: 108-112.

Trivedi SG, Newson J, Rajakariar R, Jacques TS, et al. (2006). Essential role for hematopoietic prostaglandin D2 synthase in the control of delayed type hypersensitivity. Proc. Natl. Acad. Sci. U. S. A. 103: 5179-5184.

Wall ME, Rechtsteiner A and Rocha LM (2003). Singular value decomposition and principal component analysis. In: A practical approach to microarray data analysis (Berrar DP, Dubitzky W and Granzow M, eds.). Kluwer, Norwell, 91-109. 
Wang D, Sun Y, Stang P, Berlin JA, et al. (2009). Comparison of methods for correcting population stratification in a genome-wide association study of rheumatoid arthritis: principal-component analysis versus multidimensional scaling. BMC Proc. 3 (Suppl 7): S109.

Wei P, Rong Y, Li L, Bao D, et al. (2009). Characterization of trans-neuronal trafficking of Cbln1. Mol. Cell Neurosci. 41: 258-273.

Wolc A, Arango J, Settar P, Fulton JE, et al. (2012). Genome-wide association analysis and genetic architecture of egg weight and egg uniformity in layer chickens. Anim. Genet. 43: 87-96.

Xie L, Luo C, Zhang C, Zhang R, et al. (2012). Genome-wide association study identified a narrow chromosome 1 region associated with chicken growth traits. Plos One 7: e30910.

Yan LX, Wu QN, Zhang Y, Li YY, et al. (2011). Knockdown of miR-21 in human breast cancer cell lines inhibits proliferation, in vitro migration and in vivo tumor growth. Breast Cancer Res. 13: R2. 